\title{
The criteria of rational medical examination
}

\author{
Jinguo Wang $^{1, \mathrm{a}}$ and Na Wang ${ }^{2, \mathrm{~b}^{*}}$ \\ ${ }^{1}$ the First Hospital of Jilin University, Changchun 130021, China; \\ 2 the First Hospital of Jilin University, Changchun 130021, China \\ awangjinguolily@163.com, 'wangna080613@163.com
}

Keywords: Medical examination, rationality, criteria.

\begin{abstract}
The connotation of medical check rationality is quite complicated, and its evaluation standards must be multidimensional and multifaceted. Whether the cognizance of the medical examination is reasonable or not is not the proliferation of personal subjective random and emotions. From a certain extent, the rationality of the medical examination evaluation standards of materiality is to balance the interests of the subject of medical examination related a strategy or structure, not only embodies the government of our country health enterprise nature and the development goal of positioning, both doctors and embodies the main body to compete for power in the struggle for their own interests, but also implies the social economic benefit allocation mechanism and the concept of health interests of different social groups.
\end{abstract}

\section{Introduction}

Medical examination technology is one of the medical technology, medical science and other areas of science and technology and the combination of the product of applied to medical practice. Medical examination by medical inspection equipment operation technology and medical examination behavior of medical information processing technology of two parts. The former is referred to as the object of medical examination technology elements, and can be referred to as the medical check material technology. The latter called medical examination technology main body elements, and can be referred to as the medical check technical ability. Medical examination of these two kinds of technology are not isolated, but rather complementary, technician have medical knowledge, experience, skill and skill is reflected through the materials such as medical equipment technical conditions, by the same token, medical check material technology need through the operation of the medical personnel and medical information processing skills can play their role, the same material will technical conditions for the medical staff of technical ability of different clinical value has very big difference. Technical rationality of medical examination evaluation should, therefore, to distinguish the material technology is reasonable and the technical ability reasonable two levels, according to two different levels corresponding technical features and technical specification requirements, respectively, with specific and comprehensive judgment of argument, and in this way can the rationality of the evaluation is objective and correct.

\section{Text}

Countries in order to better protect and promote health of residents, made a number of mandatory and normative health technology assessment and access regulations and policies. Admittance of medical examination technology refers to the relevant experts through safety and effectiveness of medical examination technology, economy and social adaptability of the ethical aspects after evaluating system, in accord with certain standard rear allow applied in patients with diagnosis and treatment of medical institutions service procedures and provisions. Medical examination technology clinical access should follow the scientific, safe, standardized, effective, economic and ethical principles. By the medical institution for the safety, efficacy precise medical examination technique for clinical application of conventional management, by the administrative departments of public health safety, 
efficacy precise but involves certain ethical problems or risk control management of higher medical examination technology, involving major ethical issues, high risk and require the use of scarce resources, safety and effectiveness is still subject to standardize clinical trials to further verify the medical examination technology needs to be strict control on the administrative departments for public health.Article 12 of these measures also emphasized the medical institutions to carry out clinical inspection items must be released by the ministry of health grant to carry out clinical inspection items [1]. In general, the admittance of medical examination technology including exploratory admittance of medical examination technology, restricted medical inspection technology application access, the promotion of medical examination technology application access three, each type of access standards and requirements are different, the former yan in the latter, and with the development of medical technology matures and, the existing technical access standards for medical examination should be timely adjust.

To determine the evaluation standards of rationality of technology, the medical examination should adhere to the following basic principles: should distinguish between the medical inspection technology of different types and corresponding access requirements, for different medical examination technology projects should not only have a common evaluation principles, but also reflects the differences in evaluation of technical indicators, to explore medical inspection technology and restrictive medical inspection shall request a higher evaluation. Medical examination evaluation standards of technical rationality should be due to the different historical periods and different parts of the different and have different social and economic development level. Medical examination technology rationality evaluation standards should not only is helpful to promote the development of medical science and medical technology progress and improve the quality of medical treatment, and is beneficial to patients' medical security, technical security of medical examination is the first factor of evaluation of this technology is reasonable, is the first line of defense to protect patients' rights.Medical check the rationality of the evaluation criteria included not only the security of medical examination technology,

Should also include the effectiveness of clinical effect, economy and social ethics, adaptability, etc., among them, the validity refers to the medical inspection efficiency, effectiveness and sensitivity of;Economy refers to the cost-effectiveness and cost-benefit, the rationality of the cost - effectiveness analysis. Social adaptability refers to a specific time and space under the medical examination of social, legal, ethical, and political conditions of anastomosis with the tacit understanding.

In addition, the medical check the rationality evaluation to avoid the impact of medical technology, a medical examination of technology is the medical check service as a purely technical activities, played down the humanity quality and medical ethics accomplishment of medical examination behavior, played down the patient's personality and human nature, and ignore the emotional communication between doctor and patient, ignored the value concept of patients is the medical service center, thus the one-sided pursuit of sophistication and automation, medical technology, the pursuit of medical inspection technology of profit maximization, makes original noble purpose of medical examination provincial and mechanization, at the same time also can cause medical technology limited resources of the waste and waste.

Some scholars think that: "in the west, the legitimacy of the indicates that something has been acknowledged and recognized and accepted, understandable that people from the heart for the political order, authority and power, the country, the government and social groups such as trust, support and approval; in China, most scholars believe that the legitimacy refers to a particular object is the current national legal norms, whoever meets the constitution and the rules of law, is legitimate, is legal." [2] Some scholars also put forward, legitimacy refers to the act of people pursue and realize their own interests conform to the requirements of the current law values and norms.

In recent years, the legal issues have gradually become a hot issue of China's academic circles, more and more scholars begin to target to the legitimacy study of many specific problems [3]. Law is the product of human highly rational, use legal rules to regulate people's behavior is the inevitable requirement of society ruled by law, and also the embodiment of social progress.Because the law with the procedural law and substantive law, public law and private law different categories, such as 
legitimacy has procedure legitimacy and the legality of the essence, form validity and legitimacy of different level, such as the difference between the entity and, therefore, in terms of legitimacy itself, is indeed a connotation is very rich and controversial concept.

From the law perspective, medical examination behavior is a kind of special legal act, is medical legal act. Medical practice is divided into special medical legal behavior and general medical legal act, think the special medical legal act refers to every treatment, correction or to prevent human disease, injury disability or health care for direct purpose of the examination, diagnosis, and treatment or based on the results of examination, diagnosis, treatment or for the purpose of the prescription drug behavior such as one or all of the floorboard of;Purpose and generalized medical legal behavior including diagnosis and treatment of medical legal act, has no therapeutic medical legal behavior, experimental medical legal behavior and aggressive medical legal act [4]. The scholar thinks, medical behavior of medical law behavior also can be divided into legal and illegal medical practice, those allowed by current law, accept or give positive behavior is the legal medical behavior, or for illegal behavior law. Both doctors at the same time, according to the medical behavior in the process of implementing whether there is a mutual intention, legal medical behavior can be divided into legal behavior and medical fact that behavior. And scholars believe that, regardless of the medical behavior itself is legal or not, if have a positive effect of law, and what legal effect, does not affect the establishment of the medical behavior, this is just a national law to evaluation results.

Guidance is in the rule of law society, law, constraints, and the evaluation of medical behavior important specification. According to China's relevant laws, regulations, and department of health rules and regulations, legal medical examination behavior should include the implementation of medical examination of the qualification of legal medical institutions, practitioners of subject qualification, legal, medical examination project and technical process of legal, medical examination behavior is not in violation of the law, social public interests, etc [5]. With the rule of law in our country policy of gradually implement, medical checks the have to accept perfect.this specification and regulating medical behavior, medical check the rationality of the evaluation also can not get away from the legitimacy of natural survey and inspection. A certain extent, the only legitimate medical examination technology and medical examination behavior might is reasonable, the rationality and legitimacy of the medical examination are the two interrelated, mutual penetration and mutual evaluation perspective of regulation.Such as defensive or excessive medical behavior, medical personnel to use forced urgent mentality health maintenance or repair, or lack of patients using medical information and their own professional and technical authority, inducing or misleading use many of the patients with no necessary or reference value for clinical diagnosis and treatment of medical examination, the medical examination is clearly unreasonable, at the same time, due to the defensive or excessive medical behavior to bring economic benefits to patients and belongs to the civil law of civil fraud, cause the patient's physical and mental health hazards also belong to intentionally hurt others the civil or criminal infringement of the body, or excessive defensive medical behavior clearly violates the provisions of general legal obligations related law in our country, is illegal, nature is not reasonable.

From a legal perspective of the rationality of the medical tests, one is to pay attention to both pursue the results of medical examination behavior motivation and purpose is good, attaches great importance to the medical examination is the behavior of the process and means proper. Three is both leave sufficient legal space for medical examination technology development, and to respect the basic rule of medical research and technological development, abide by the rules and regulations of the state adjust medical examination behavior.

Medical ethics is a special kind of professional ethics, is the embodiment of the social general ethics in the field of medicine, through specific medical ethics and code of ethics to influence and restriction of medical staff, adjustment between doctor and patient, between medical staff and the relationship between medical staff and society.Medical check the rationality of the evaluation must penetrate and holds many medical ethics and the basic requirements of the code of conduct, its rationality and ethical morality has a strong accommodation and complementarity, as you can imagine, is not in conformity with the ethics of medical examination may not be for reasonable 
evaluation.Medical ethics for medical behavior should be respect, do not harm, benefit and justice four fundamental ethical principles [6]. In medical examination practice, we need to respect patients' personal dignity, privacy and the right of informed consent, understand the patient's medical examination requirements will, it is fair to treat every patient, should earnestly put the patient's physical and mental health benefits and economic benefits as medical examination work the first goal and the primary indicators of quality evaluation.

Work discipline, serious and responsible to each patient or samples to equality, to guarantee the specification of the medical check process and check the result accuracy; third, medical institutions in the introduction and development of new medical check the project, the patients' medical check consumption desire and ability to cope with an in-depth investigation and research, from the perspective of ethics to science and medical technology assessment. At the same time, must strengthen the medical check quality consciousness, strengthening the professional ethics education of medical staff, set up the mechanism of doctor-patient communication, correctly guide the patient's medical examination requirements, the rationality of idea and ethics standard properly combined and implementation of each link to medical examination.

\section{Summary}

Medical check rationality evaluation standards should not only consider the medical staff to implement medical examination behavior of professional ethics and professional skills of effective regulation, and the patient's health and other lawful interests of adequate protection, also want to consider the operation rules of the health care industry as well as correlation with the interests of the other industries such as medium, but also by China's political, economic and cultural development and other macroeconomic indicators.

\section{References}

[1] Xianxiang Chen,Zhen Fang,Ren Ren,Shanhong Xia,Yangmin Qian,Huaiyong Li,Lili Tian,Wenxi Zhai,Weibing Xu,Hui Gao. Simple and efficient baseline removal method for a smartphone based ECG detection device [A]. Proceedings of 2013 International Conference on Future Computer and Information Technology (ICFCIT 2013) [C]. 2013

[2] Fang Qingwei. Research on Evaluation Index System of Management Effectiveness on Hospital Human Resource Based on Balanced Scorecard [A]. Proceedings of 2011 International Conference on Fuzzy Systems and Neural Computing (FSNC 2011 V2) [C]. 2011

[3] Thepparit Sinthamrongruk,Napat Harnpomchai. Determination of optimal number of doctors and appointment interval for an appointment [A]. Proceedings of the International Conference on Information Engineering and Education Science [C]. 2014

[4] Ying Han, Yanyan Shi. Research on the Teaching Design of the Flipped Classroom Based on the Automobile Insurance Claim [A]. Proceedings of 2nd International Conference on Social Science and Higher Education (ICSSHE 2016 V53) [C]. 2016

[5] Linnan Cui. Rethinking of the Overall Planning for Urban and Rural Residents' Medical Insurance System [A]. Proceedings of the 2016 International Conference on Judicial,Administrative and Humanitarian Problems of State Structures and Economical Subjects (JAHP 2016) [C]. 2016

[6] Pengcheng Han,Xubin Li,Chaoyi Guo. Research on the Combination of P2P Network Credit and Insurance Based on Game Theory [A]. Advances in Social Science, Education and Humanities Research ( Volume 70) [C]. 2016 